\title{
Dapagliflozin: an insulin-independent, therapeutic option for type 2 diabetes mellitus
}

D apagliflozin-a drug that improves glycemic control by increasing urinary excretion of glucose-is both safe and effective for the treatment of type 2 diabetes mellitus (T2DM), according to the findings of two multicenter, randomized, placebo-controlled, phase III trials published recently.

Maintenance of glycemic control is a priority in the management of T2DM because chronic hyperglycemia contributes to the microvascular and macrovascular complications associated with the disease. However, first-line approaches such as lifestyle management and treatment with metformin often become insufficient to provide adequate glycemic control over time.

"Most patients will require a combination of differently acting agents as the severity of the disease advances, and even then it is difficult to maintain blood glucose control in many patients," points out Clifford J. Bailey of Aston University, Birmingham, UK, a lead researcher of one of the two trials. "Additional bloodglucose-lowering therapies are needed that can act independently of the deteriorating insulin status and assist weight loss."

Dapagliflozin acts in an insulinindependent fashion, and as such, holds potential to be used throughout the natural history of the disease. This agent is a highly selective inhibitor of the sodium-glucose cotransporter 2 , a glucose transporter located in the proximal tubule of the kidney nephron that acts to reabsorb most of the glucose filtered by the glomeruli. By prevention of this reabsorption, dapagliflozin enhances glucose excretion in the urine and, thereby, reduces hyperglycemia and increases urinary loss of calories.

Bailey and co-researchers assessed the efficacy and safety of dapagliflozin in 546 adult

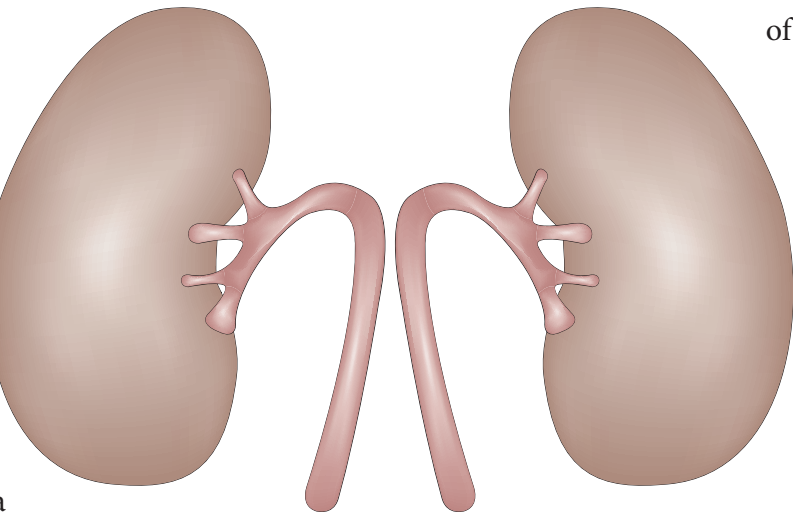

of the study by Bailey et al., mean reduction in $\mathrm{HbA}_{1 \mathrm{c}}$ from baseline was significantly greater in the $5 \mathrm{mg}$ and $10 \mathrm{mg}$ treatment groups than the placebo group. Furthermore, dapagliflozin was efficacious in $\mathrm{HbA}_{1 \mathrm{c}}$ reduction in an additional cohort of 73 patients with $\mathrm{HbA}_{1 \mathrm{c}}$ levels of 10.1-12.0\% at recruitment. Although the researchers observed only a nonsignificant trend for greater weight loss in the dapagliflozintreated than the placebo groups, they highlight that the placebo group lost considerable weight owing to diet and exercise counseling during the trial.

Overall, dapagliflozin was safe and well-tolerated in patients with T2DM. Nevertheless, a higher proportion of patients treated with dapagliflozin than placebo in the trials reported genital infections and/or urinary tract infections; therefore, the researchers suggest vigilance and standard approaches to swiftly treat these conditions that could be related to elevated glucose concentrations in the urine.

Results of long-term studies will be eagerly awaited as "this approach is a new option to improve glycemic control that can be used as monotherapy or in conjunction with any of the other therapies independently of insulin status," concludes Bailey.

\section{Carol Wilson}

